\title{
Les Conservateurs écossais et les élections de 2017 à la Chambre des Communes : succès éphémère ou détoxification durable?
}

The Scottish Conservatives and the 2017 General Election: Ephemeral Success or Durable Detoxification?

\section{Edwige Camp-Pietrain}

\section{OpenEdition}

\section{Journals}

Electronic version

URL: http://journals.openedition.org/rfcb/2135

DOI: $10.4000 /$ rfcb. 2135

ISSN: $2429-4373$

Publisher

CRECIB - Centre de recherche et d'études en civilisation britannique

\section{Electronic reference}

Edwige Camp-Pietrain, «Les Conservateurs écossais et les élections de 2017 à la Chambre des

Communes : succès éphémère ou détoxification durable ? ", Revue Française de Civilisation Britannique [Online], XXIII-2 | 2018, Online since 14 September 2018, connection on 02 May 2019. URL : http:// journals.openedition.org/rfcb/2135; DOI : 10.4000/rfcb.2135

This text was automatically generated on 2 May 2019.

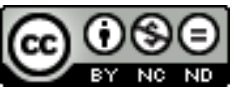

Revue française de civilisation britannique est mis à disposition selon les termes de la licence Creative Commons Attribution - Pas d'Utilisation Commerciale - Pas de Modification 4.0 International. 


\title{
Les Conservateurs écossais et les élections de 2017 à la Chambre des Communes : succès éphémère ou détoxification durable?
}

\author{
The Scottish Conservatives and the 2017 General Election : Ephemeral Success or \\ Durable Detoxification?
}

Edwige Camp-Pietrain

\section{Introduction}

1 Le 8 juin 2017, en emportant 13 des 59 sièges écossais à la Chambre des Communes, les Conservateurs écossais ont mis un terme à leur déclin. En effet, alors que le Parti unioniste écossais constituait le deuxième parti écossais dans l'après-guerre, son intégration au Parti conservateur britannique (à partir de 1965), puis son association aux politiques néolibérales des gouvernements de Margaret Thatcher et de John Major, perçues comme inadaptées aux besoins et aux valeurs écossais, avaient durablement altéré son image ${ }^{1}$. En 1997, assimilé à un parti anglais, le Parti conservateur avait perdu tous ses députés en Écosse (tableau 1) ; l'un de ces derniers, Malcolm Rifkind, évoqua même une marque devenue toxique ${ }^{2}$. Cette défiance s'enracina dans les années 2000, les Conservateurs écossais, sans cesse renvoyés à la poll tax ${ }^{3}$, ne parvenant à obtenir qu'un seul député à la Chambre des Communes. Ils recouvrèrent néanmoins une représentation grâce au Parlement écossais (Holyrood) créé en 1999 dans le cadre de la dévolution du pouvoir, à laquelle ils s'étaient opposés ${ }^{4}$.

2 L'inversion de tendance en 2017 est à mettre au crédit de Ruth Davidson, leader des Conservateurs écossais depuis 2011. Toutefois, celle-ci a également bénéficié du travail de son prédécesseur, Annabel Goldie, et de divers événements, notamment le référendum d'autodétermination de 2014, véritable tournant dans la vie politique écossaise. Elle a su 
les exploiter au profit de son parti. Cet article se propose d'étudier les conditions favorables aux Conservateurs écossais, tant dans les mois précédant le scrutin qu'au cours de la campagne, ainsi que les interrogations suscitées par la portée de ce succès relatif jusqu'au début de l'année 2018. L'objectif est de tenter de déterminer si une réelle détoxification est en cours.

\section{Un contexte porteur}

Les Conservateurs écossais ont accueilli avec enthousiasme l'annonce de cette élection anticipée car ils disposaient de nombreux atouts. Tout d'abord, ils étaient dotés d'un véritable leader à la suite de diverses réformes entamées dès les débuts de la dévolution. En 1998, la commission Strathclyde avait rationalisé la structure du parti, dirigé par un chairman, dont la nomination devait toutefois être approuvée par le leader britannique du parti, et par un vice-président élu par les adhérents ${ }^{5}$. Cependant, les deux leaders successifs des Conservateurs écossais, David McLetchie puis Annabel Goldie (à partir de 2005), n'étaient que les chefs du groupe de députés à Holyrood. De plus, ils avaient émergé sans devoir affronter de concurrent. Par conséquent, en 2010, prenant acte de l'importance croissante du Parlement écossais dans la vie politique locale, Goldie instaura une commission, dirigée par Lord Sanderson, qui proposa la création d'un leader du parti en Écosse, élu directement par les membres, à l'issue d'un scrutin effectivement disputé6.

Ces nouvelles dispositions prirent effet après les élections au Parlement écossais de 2011, lorsque Goldie démissionna. Murdo Fraser, député expérimenté, préconisait un Parti conservateur écossais distinct, sur le modèle de la CSU bavaroise, étroitement liée à la CDU. Il militait également en faveur d'une autonomie fiscale complète pour le Parlement écossais, ce dernier, en charge de tous les impôts collectés en Écosse, devenant ainsi pleinement responsable de ses décisions. Face à lui, Davidson, qui venait d'entrer à Holyrood après avoir travaillé dans les médias audiovisuels, apparaissait comme la candidate du statu quo, tant sur le plan des statuts du parti que s'agissant des réformes constitutionnelles. Toutefois, par sa personne même, Davidson incarnait un changement : elle avait fréquenté un collège public et ne faisait pas mystère de son homosexualité ${ }^{7}$. Sous d'autres aspects, elle rassurait aussi, en tant que fidèle de l'Église d'Écosse et réserviste de l'armée. C'est elle qui l'emporta.

Ensuite, les Conservateurs écossais devaient démontrer leur utilité dans la vie politique. S'agissant du programme, dès 1998, la commission Rifkind avait préconisé des politiques écossaises dans les domaines dévolus; mais ses propositions s'étaient heurtées à la structure du parti et à un manque de volonté ${ }^{8}$. En ce qui concerne les actions, dès 2007, profitant de l'avènement d'un gouvernement SNP minoritaire, Goldie lui avait apporté son soutien lors du vote du budget annuel, en échange de concessions en matière fiscale (baisse ou suppression, au profit des PME, de la taxe sur les entreprises prélevée par les collectivités territoriales). Cet appui, essentiel pour le maintien au pouvoir du First Minister Alex Salmond, avait cessé en 2011, lorsque le SNP avait obtenu une majorité absolue de sièges.

6 Les Conservateurs ont su tirer avantage des débats constitutionnels engendrés par les gouvernements SNP. Alors que son parti s'était tenu à l'écart des projets de dévolution jusqu'en 1997, Goldie souhaita le replacer au centre de la vie politique écossaise en le faisant participer à la commission multipartite Calman dont les recommandations inspirèrent le Scotland Act voté à Westminster en avril 2012. Cette loi accroissait les 
pouvoirs fiscaux de Holyrood, avec un pouvoir de modulation des taux de l'impôt sur le revenu. Ensuite, Davidson se lança dans la campagne contre l'indépendance de l'Écosse, n'hésitant pas à se réapproprier le terme unioniste. Qui plus est, alors qu'elle se déclarait satisfaite de la loi de 2012, sa position évolua sous l'impulsion de David Cameron. Le Premier ministre reconnaissait que le SNP disposait de la légitimité nécessaire pour organiser son référendum d'autodétermination. En l'autorisant, il comptait le gagner aisément, tout en accordant plus d'autonomie à l'Écosse. Par conséquent, Davidson accepta de nommer une commission, sous la tutelle de Lord Strathclyde, chargée de formuler des propositions visant à accroître les pouvoirs du Parlement écossais. En mai 2014, celle-ci recommanda la dévolution de certaines allocations, mais aussi des taux et des tranches de l'impôt sur le revenu, impôt le plus visible pour les contribuables et le plus important pour les finances publiques ${ }^{9}$. Davidson endossa cette position, jusque-là minoritaire au sein $d u$ parti $^{10}$, et plus avancée que celle des Travaillistes (lesquels prônaient alors une extension du modèle retenu en 2012), afin de montrer que le rejet de l'indépendance n'était pas synonyme de statu quo.

7 L'engagement à poursuivre la dévolution, assorti d'un calendrier, fut repris par les leaders des trois partis britanniques peu avant le scrutin. Au lendemain de ce dernier, Cameron instaura une commission multipartite (intégrant aussi les indépendantistes). Cette commission Smith, au sein de laquelle les Conservateurs étaient représentés par Goldie (désormais membre de la Chambre des Lords) et Adam Tomkins, juriste, retint nombre de leurs propositions, notamment au plan fiscal. Le projet fut entériné par le Scotland Act voté à Westminster en 2016. Les Conservateurs se retrouvaient ainsi au cœur de la vie politique écossaise.

Ils confirmèrent ce positionnement lors du référendum sur le maintien du Royaume-Uni dans l'UE du 23 juin 2016. Davidson, attachée à ce maintien, put compter sur le soutien de la majorité de ses députés au Parlement écossais, un mois avant le scrutin ${ }^{11}$. Cela contrastait avec les déchirements du parti en Angleterre. Davidson fut d'ailleurs sollicitée pour prendre part à la campagne britannique et porter la contradiction à Boris Johnston. L'immigration, l'un des principaux arguments des partisans du Brexit, ne constituait pas un enjeu en Écosse, du moins pour les partis politiques qui s'accordaient sur la nécessité de l'encourager pour des raisons économiques et éthiques.

9 Enfin, forts de ce positionnement en faveur de l'UE et de la poursuite de la dévolution, tout en restant hostiles à l'indépendance de l'Écosse, les Conservateurs avaient connu une progression substantielle à chaque scrutin, parvenant à doubler leurs proportions de voix (tableau 2). Aux élections au Parlement écossais de mai 2016, ils avaient, pour la première fois, devancé le Parti travailliste en tant que deuxième parti ; de plus, sur 32 députés, 7 avaient emporté des circonscriptions, au scrutin uninominal (les autres étant élus sur des listes régionales, à la proportionnelle). Aux élections dans les collectivités territoriales unitaires de mai 2017, fondées sur un scrutin proportionnel, ils avaient obtenu un quart des suffrages.

10 Les intentions de vote pour les élections à la Chambre des Communes s'inscrivaient dans cette dynamique ${ }^{12}$. Certes, le résultat des Conservateurs aux élections législatives de mai 2015 était proche de ceux des scrutins antérieurs. Mais le SNP ayant, fait inédit, emporté la quasi-totalité des sièges écossais, les intentions de vote en faveur des Conservateurs ont alors dépassé celles en faveur des Travaillistes, les premiers paraissant plus susceptibles de s'opposer au parti indépendantiste. Au début de l'année 2017, l'écart entre les deux partis s'était resserré, mais les Conservateurs écossais restaient en tête. 
Davidson, portée par ces sondages, et par les résultats antérieurs, était relativement sereine lorsque le Premier ministre, Theresa May, annonça des élections anticipées à la Chambre des Communes.

\section{Une campagne ancrée en Écosse}

11 Les Conservateurs ont axé leur campagne sur les enjeux écossais, ciblant leur électorat traditionnel.

Davidson, qui s'était affirmée pendant les campagnes référendaires de 2014 et 2016, était devenue un réel atout pour son parti. Elle était plus appréciée que Theresa May et Kezia Dugdale, leader des Travaillistes écossais, et faisait jeu égal avec Nicola Sturgeon, First Minister SNP depuis novembre 2014 (tableau 3). Elle avait même un avantage sur cette dernière car elle recueillait moins d'opinions franchement défavorables étant donné qu'elle n'avait pas à gouverner. Cette avance était précieuse, car ce sont les leaders écossais qui ont mené la campagne, même s'ils n'étaient pas candidats.

Davidson sut exploiter cette popularité pour dicter l'ordre du jour de la campagne. Elle a imposé des sujets écossais face aux conseillers du Premier ministre qui lui enjoignaient de faire la promotion d'un gouvernement britannique "fort et stable " pour défendre les intérêts britanniques dans le cadre du Brexit ${ }^{13}$. De plus, elle a contré les intentions de Sturgeon, visant à profiter du contraste entre le gouvernement écossais (désireux de formuler des propositions réalistes pour conserver la place de l'Écosse dans l'UE) et le gouvernement britannique (engagé à faire sortir l'ensemble du Royaume-Uni du marché unique et de l'union douanière, sans anticipation). Davidson a orienté la campagne autour $\mathrm{du}$ refus du second référendum d'autodétermination que la First Minister avait formellement réclamé en mars, avant l'annonce des élections anticipées, afin d'échapper à ce hard Brexit ${ }^{14}$. Or, si l'UE pouvait constituer un thème rassembleur en Écosse, la perspective d'un second débat sur l'indépendance était source de divisions. Elle était exclue par les unionistes (mais les Conservateurs étaient plus unis sur ce point que les Travaillistes), tandis que nombre d'indépendantistes la jugeaient prématurée. Elle était plébiscitée par $40 \%$ des Écossais seulement ${ }^{15}$.

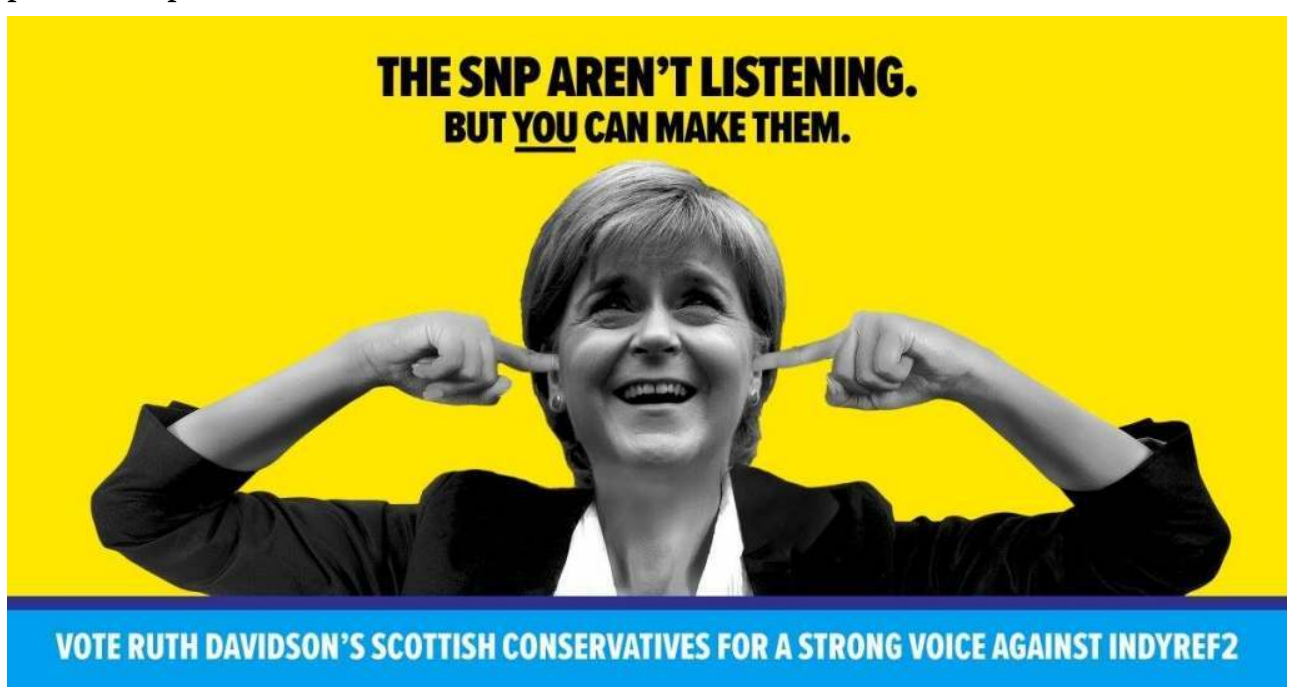

Scottish Conservative poster 2017 

marge de la vie politique écossaise, comme le montraient les débats à Holyrood pendant la campagne britannique. Tout d'abord, les Conservateurs écossais s'accommodaient du Brexit. Si dans les jours suivant le référendum de juin 2016, Davidson avait souscrit à la volonté exprimée par Sturgeon de conserver la place de l'Écosse dans l'UE, elle a repoussé les motions suivantes, au motif que la First Minister n'était pas prête à exclure un second référendum d'autodétermination. Qui plus est, ses députés ont endossé les espoirs, fréquemment exprimés par leurs collègues à Westminster, de négociations de nouveaux traités commerciaux ${ }^{16}$. controversée parmi les principaux intéressés, les pêcheurs considérant celle-ci comme une source de pillage de leurs ressources par leurs concurrents européens. Les candidats conservateurs se sont empressés de signer le texte anti-PCP de la Scottish Fishermen's Federation (SFF), pour mieux dénoncer la position pro-européenne du SNP.

Certes, en décembre 2016, dans son Livre blanc, le gouvernement écossais avait envisagé la possibilité pour l'Écosse de rester dans le marché unique sans quitter le Royaume-Uni, sur le modèle norvégien qui exclut l'adhésion à la PCP. Mais une commission interne au Parti conservateur écossais a écarté cette perspective en février $2017^{17}$. Les députés conservateurs n'ont cessé de marteler que le marché britannique était quatre fois plus important pour l'Écosse que le marché intérieur de l'UE. étaient tenus pour responsables des politiques du gouvernement May, notamment la limitation du crédit d'impôts à deux enfants - sauf si le troisième résultait d'un viol, dûment attesté. En dépit de ses réserves personnelles, Davidson a défendu cette réforme au nom de la saine gestion des finances publiques ${ }^{18}$. Cet épisode, largement médiatisé, contrariait son travail de détoxification. En outre, à cette époque, le Parlement écossais discutait de la création de ses propres allocations, autorisée par le Scotland Act de 2016. Or, le gouvernement Sturgeon entendait instaurer une Sécurité sociale écossaise, centrée sur les besoins des allocataires et non sur les évaluations obligatoires, assorties de sanctions, pour mieux se démarquer des politiques britanniques. Les Conservateurs écossais ont tenté de justifier celles-ci, en rappelant que l'objectif était de faciliter le retour à l'emploi des allocataires, tout en mettant l'accent sur les coûts de la réforme écossaise ${ }^{19}$.

Dans le même temps, Davidson et ses collègues ne pouvaient rester totalement à l'écart $\mathrm{du}$ consensus écossais relatif à l'universalité de certaines prestations. Dans leur programme, ils se sont mis à soutenir la gratuité des médicaments, en vigueur depuis 2010 en Écosse, mais exclue en Angleterre, ainsi que le maintien du versement généralisé de l'allocation liée aux dépenses de chauffage des personnes âgées, sur le point d'être abandonné en Angleterre ${ }^{20}$. Ils ont dénoncé le creusement des inégalités, continuant à faire de la lutte contre ces dernières un objectif des politiques publiques, s'agissant notamment des enfants. Ces divergences territoriales, reflets de l'esprit de la dévolution, n'ont pas suscité de débat au sein du parti.

Enfin, les Conservateurs écossais paraissaient plus à l'aise sur la fiscalité. Selon eux, les impôts écossais ne devaient pas dépasser les impôts levés en Angleterre, même si cela soulevait la question de la pertinence de leur dévolution. Ils ont donc critiqué la modeste réforme de l'impôt sur le revenu (moindre revalorisation du seuil d'assujettissement au taux de $40 \%$ que dans le reste du royaume) tout en se félicitant du projet de réduction de 
la taxe sur les passagers aériens ${ }^{21}$. Cependant, ils n'ont pas voté le budget du gouvernement Sturgeon, minoritaire depuis 2016, car la vie politique écossaise était réorganisée selon un clivage unioniste-indépendantiste.

Le 8 juin 2017, à l'issue de cette campagne efficace, les Conservateurs écossais ont rassemblé 757949 suffrages, contre 400000 environ dans les années 2000. Ils ont doublé leurs parts de voix, de $14,5 \%$ en 2015 à $28,6 \%$, réalisant leur meilleur score depuis le début des années quatre-vingt (tableau 1). Leurs résultats ont progressé dans 48 des 59 circonscriptions écossaises. Le nombre de sièges dans lesquels ils ont recueilli plus de $30 \%$ (seuil pouvant laisser espérer une victoire) est passé de 4 à 20 (tableau 4). À l'inverse, ils ont enregistré des scores inférieurs à 10\% dans un seul siège, contre 24 en 2015.

Les Conservateurs, qui comptaient un seul député depuis 2001, en ont obtenu 12 autres, résultat inédit depuis le début des années quatre-vingt-dix. De plus, ils ont récupéré les sièges qu'ils représentaient alors, au nord-est (Aberdeen South, Aberdeenshire West \& Kincardine, Angus, Banff \& Buchan, Gordon, Moray), au centre (Ochil \& South Perthshire, Stirling, Ayr, Carrick \& Cumnock, East Renfrewshire), et au sud (Berwickshire, Roxburgh \& Selkirk, Dumfries \& Galloway) où ils avaient déjà un siège (Dumfriesshire, Clydesdale \& Tweeddale) depuis $2005^{22}$. Cela résultait d'un travail de remobilisation de leur électorat, entrepris au début des années 2000 dans leurs anciens fiefs, en dépit des redécoupages ${ }^{23}$. Leurs objectifs, clairement identifiés, faisaient l'objet d'une attention constante. Ainsi, les sièges dans lesquels leurs dépenses électorales excédaient $90 \%$ des plafonds autorisés étaient quasiment les mêmes qu'en 2010 (tableau 5).

Tous ces sièges ayant un député SNP depuis 2015, les Conservateurs ont battu des personnalités du premier parti d'Écosse, tels Alex Salmond, artisan du référendum de 2014, ou des députés entrés au Parlement avant la vague nationaliste de 2015, tels Angus Robertson (Moray), numéro deux du parti, Mike Weir (Angus) ou Eilidh Whiteford (qui avait succédé à Salmond à Banff).

Les Conservateurs écossais ont tiré avantage de plusieurs facteurs. Tout d'abord, sur le plan institutionnel ${ }^{24}$, ils ont recueilli les suffrages de près de la moitié des personnes ayant voté contre l'indépendance en 2014, qu'ils avaient explicitement ciblées ${ }^{25}$. Mais ils ont également attiré près de $50 \%$ des électeurs pro-Brexit, contre seulement un tiers en mai 2016, alors même que Davidson a indiqué qu'elle voterait à nouveau en faveur du maintien dans l'UE en cas de nouveau référendum. Ensuite, sur le plan sociologique, le parti a renoué avec son électorat traditionnel (tableau 6). Mais, à l'exception des personnes les plus âgées, il est toujours largement devancé par le SNP dans toutes les tranches d'âge et les classes sociales. Enfin, sur le plan territorial, les coefficients de corrélation montrent une continuité avec les résultats de 2015 (tableau 7). Le parti a progressé dans les sièges lui étant les plus favorables, tels ceux du nord-est, comptant des communautés de pêcheurs eurosceptiques, tendance que Weir et Whiteford n'ont pu contrer en signant le document anti-PCP de la SFF.

En outre, les députés conservateurs écossais se rapprochent davantage de leurs électeurs que ceux des années quatre-vingt-dix. Seuls 2 sur 13 ont fréquenté une école privée, coûteuse et sélective (ils étaient 6 sur 11 en 1992), contre $45 \%$ de l'ensemble des députés conservateurs élus en $2017^{26}$.

Il leur reste désormais à démontrer le rôle qu'ils peuvent jouer à la Chambre des Communes. 


\section{Un succès relatif, semé d'embûches} structure de l'économie écossaise. Ils ont ainsi rappelé l'importance de la pêche pour les communautés qu'ils représentent. Or les pêcheurs écossais estiment avoir été trahis par tous les dirigeants conservateurs, qu'il s'agisse de Heath lors de l'entrée dans la CEE (accès des bateaux européens à la zone économique exclusive britannique de 200 milles nautiques), de Thatcher (instauration de quotas) ou de Major (démantèlement d'une partie de la flotte). May pourrait avoir la même tentation pour obtenir un accord de libreéchange avec l'UE à l'issue du Brexit car le secteur est marginal à l'échelle britannique. Elle a déjà avalisé le maintien du Royaume-Uni dans la PCP pendant les deux années de transition suivant la sortie formelle de l'UE, en contradiction avec la promesse faite aux pêcheurs $^{29}$. Par ailleurs, toutes les projections montrant que seule l'immigration peut enrayer le déclin de la population écossaise, les Conservateurs écossais ont tenté, lors des auditions de la commission des Affaires écossaises, de cerner les moyens de répondre aux besoins locaux en s'affranchissant des politiques restrictives envisagées par le gouvernement britannique ${ }^{30}$. Mais ils préfèreraient opter pour des dérogations sectorielles, valables pour l'ensemble du Royaume-Uni.

Qui plus est, le Brexit va affecter la dévolution du pouvoir à l'occasion de son vingtième anniversaire. Selon le projet de loi déposé en juillet 2017 (EU (Withdrawal) Bill), le gouvernement britannique doit, dans un premier temps, récupérer toutes les compétences exercées à Bruxelles, même dans les domaines dévolus (agriculture, pêche), avant de confier au Parlement écossais certaines d'entre elles, à définir. Pour le gouvernement SNP, il s'agit d'une re-centralisation, inacceptable en l'état. Face à ces tensions, susceptibles de déboucher sur une crise constitutionnelle, les Conservateurs écossais se sont érigés en défenseurs de la dévolution, affirmant être à l'origine du Scotland Act de $2016^{31}$, alors que cette loi est le fruit d'un travail transpartisan. Ils ont proposé leur médiation dans les pourparlers entre les gouvernements. Ainsi, à la fin de 2017, David Mundell, ministre en charge de l'Écosse au sein du gouvernement britannique, s'est engagé à publier une liste de compétences devant être dévolues à 
Holyrood. Mais ses 12 collègues écossais, peu désireux de contraindre son action, ont voté contre les amendements déposés par leurs adversaires écossais ${ }^{32}$.

31 Les Conservateurs écossais peinent également à trouver une voix distincte sur les questions sociales, en particulier la mise en place de l'universal credit, une allocation fusionnant et plafonnant six prestations. Les députés SNP et travaillistes, indignés de retards de versements responsables d'une explosion des recours aux banques alimentaires, ont appelé à une pause. Mais les Conservateurs écossais ont soutenu les aménagements modestes apportés par le gouvernement May (réduction du délai précédant le premier versement, gratuité des appels téléphoniques), soulignant que seul le retour au travail pouvait rendre leur dignité aux allocataires ${ }^{33}$.

32 À la fin du mois de novembre, lors de la présentation du projet de loi de finances, c'est le gouvernement britannique lui-même qui a mis en avant la force de conviction des Conservateurs écossais, à l'origine de la restauration de l'exonération de TVA des services d'urgence et de l'autorisation $\mathrm{du}$ transfert entre compagnies pétrolières des dégrèvements compensant les coûts du démantèlement des plates-formes en mer du Nord 34 .

Au Parlement écossais, les Conservateurs écossais, dans l'opposition, ne sont pas tenus d'observer la même discipline. Les périodes électorales étant terminées, ils retrouvent une liberté de parole afin de promouvoir les intérêts écossais. S'agissant des questions institutionnelles, Davidson a évoqué en juin un Brexit "ouvert», invitant le gouvernement britannique à tenir compte des intérêts économiques, en privilégiant l'accès au marché européen au lieu de se focaliser sur l'immigration et ce, pour l'ensemble du royaume ${ }^{35}$. En décembre, elle s'est élevée contre l'accord spécifique à l'Irlande du Nord que semblait envisager May, contraignant celle-ci à opter pour un alignement réglementaire de l'ensemble du Royaume-Uni qui pourrait se rapprocher d'un soft Brexit ${ }^{36}$. Quant aux questions écossaises, Davidson reste vigilante au sujet du second référendum d'autodétermination car le report annoncé par Sturgeon en juin ne vaut pas renonciation. Elle accuse le SNP d'avoir consacré ses dix années au pouvoir à l'indépendance de l'Écosse au lieu de gouverner. Cependant, elle est en retrait sur la question de la dévolution. En mars 2018, son groupe parlementaire s'est désolidarisé du consensus à Holyrood sur la nécessité de confier au Parlement écossais toutes les compétences rapatriées de l'UE dans les domaines dévolus ${ }^{37}$.

Davidson se mue en défenseur des services publics écossais. Ainsi, elle a salué la volonté de Sturgeon d'accorder plus de pouvoirs aux chefs d'établissements scolaires, ainsi que la suppression, pour les personnes handicapées, de la condition d'âge pour la prise en charge des frais de soins personnels liés à la dépendance (un droit réservé jusque-là aux personnes retraitées) ${ }^{38}$. Elle a même évoqué la construction de logements sociaux par les collectivités territoriales, politique abandonnée sous Thatcher. Qui plus est, les Conservateurs écossais affichent leurs préoccupations sociales. Ils ont salué le dépôt de la loi portant création d'un régime écossais de Sécurité sociale fondé sur la dignité de la personne (en réaction aux politiques britanniques) et se sont réjouis du maintien d'objectifs d'éradication de la pauvreté des enfants, abandonnés par Cameron ${ }^{39}$.

C'est surtout le volet fiscal qui devait permettre aux Conservateurs d'occuper un espace politique distinct. Dans sa loi de finances pour 2018, Sturgeon a réformé l'impôt sur le revenu, en affinant sa progressivité pour les revenus les plus modestes (création de deux tranches supplémentaires) et en augmentant les taux appliqués aux deux tranches supérieures. Or, les Travaillistes, les Libéraux-démocrates et les Verts prônaient ce 
relèvement afin de financer les services publics, à la différence des Conservateurs. Mais lors du vote final, ce sont les Conservateurs et les Travaillistes qui ont rejeté le texte ${ }^{40}$.

Néanmoins, consciente du fait que ce refus de toute hausse de la pression fiscale, s'ajoutant à celui de tout second référendum d'autodétermination, ne saurait constituer un programme en vue des élections à Holyrood de 2021, Davidson a nommé un groupe de réflexion, présidé par Lord Dunlop, ancien ministre au Scottish Office, pour lui fournir des idées afin de stimuler la croissance ${ }^{41}$.

Davidson devra toutefois continuer à apporter la preuve de l'intérêt des Conservateurs pour la dévolution. En effet, 4 des 13 députés conservateurs ont préféré Westminster à Holyrood (Mundell, Lamont et surtout Ross et Thomson qui n'y sont restés qu'un an), confirmant l'attrait exercé par le centre britannique du pouvoir sur ce parti. De plus, nombre sont ceux, au sein du parti, qui voient en Davidson un leader capable de remplacer May et lui ont réservé une ovation au congrès du mois d'octobre. Certes, l'intéressée a réaffirmé que son ambition était de devenir First Minister d'Écosse, mais sans renoncer catégoriquement à une carrière britannique, notamment si elle n'obtenait pas les résultats escomptés en 2021 à Holyrood ${ }^{42}$.

En outre, si Davidson suscite l'admiration en Angleterre, sa direction n'est pas exempte de critiques en Écosse. En 2017, deux exemples ont défrayé la chronique. D'une part, lorsqu'Ian Duncan a renoncé à son mandat de député au Parlement européen pour entrer au gouvernement (avec un siège à la Chambre des Lords), Davidson a empêché Belinda Don, numéro deux sur la liste présentée aux élections de 2014, de lui succéder ${ }^{43}$. C'est Mosheena Mobarik (ancienne présidente de CBI Scotland), troisième sur cette liste, qui est devenue députée européenne. D'autre part, à la fin de l'été, Davidson a choisi de réintégrer des membres du parti ayant tenu des propos xénophobes, au motif qu'il fallait leur laisser la possibilité de s'amender ${ }^{44}$. Mais c'est ce type d'attitudes qui avait ponctuellement empoisonné les campagnes précédentes du parti. Les défaites successives de ce dernier avaient attisé les divisions internes, ainsi que l'expression d'opinions individuelles l'éloignant du centre de la vie politique. Ce sont probablement les perspectives de progression en 2017 qui avaient fait taire les dissensions.

\section{Conclusion}

$\mathrm{Au}$ regard de leurs résultats au cours des vingt dernières années, les Conservateurs ont assurément réalisé une prouesse le 8 juin 2017. Ils ont inversé la spirale des échecs successifs dans laquelle ils s'étaient enfermés. L'écart entre les voix en leur faveur et celles se portant sur les candidats SNP est passé d'un million en 2015 à 200000 en 2017. Ils ont tiré avantage d'une image positive, insufflée et incarnée par Ruth Davidson, et de leur rejet de tout second référendum d'autodétermination qui a affaibli le SNP et perturbé les Travaillistes. Ils ont su mener une campagne écossaise, évitant les écueils de la campagne britannique.

Pour autant, si les Conservateurs ont gagné du terrain, ils n'ont pas emporté le scrutin en Écosse. De plus, ils ont progressé avec un programme qui avait peu évolué et qui n'était pas en phase avec les souhaits de la population. Or, ils doivent désormais démontrer leur utilité, tant à la Chambre des Communes où ils risquent d'être éclipsés par le DUP s'agissant des pressions sur le gouvernement May, qu'à Holyrood où ils vont devoir promouvoir leurs politiques au lieu de se contenter de manifester leur opposition. Alors 
qu'ils se réapproprient le terme unioniste dans leurs publications, ils pourraient renouer avec la conception prévalant dans le parti avant l'arrivée à sa tête de Thatcher, consistant à préserver le Royaume-Uni, tout en répondant aux aspirations de ses différentes composantes; Davidson l'a bien compris, lorsqu'elle affirme défendre les intérêts de l'Écosse et le rôle de l'État ${ }^{45}$. Maintenir la dynamique pourrait se révéler être une tâche ardue, alors que l'Écosse devrait être une des régions les plus affectées par un hard Brexit. Avant la fin de l'année 2017, les Conservateurs étaient devancés par les Travaillistes dans les intentions de vote, ce qui souligne la fragilité des résultats de 2017 dans un contexte restant toutefois très incertain ${ }^{46}$. La détoxification espérée doit encore être consolidée, notamment si le Brexit continue à exacerber les divisions entre Londres et Édimbourg.

\section{Annexes}

Tableau 1 : Résultats des Conservateurs écossais aux élections à la Chambre des Communes depuis 1955

\begin{tabular}{|l|l|l|l|l|l|l|l|l|l|l|l|l|}
\hline & 1955 & 1959 & 1964 & 1966 & 1970 & F1974 & 01974 & 1979 & 1983 & 1987 & 1992 & 1997 \\
\hline $\begin{array}{l}\text { Proportions de } \\
\text { suffrages }\end{array}$ & 50,1 & 47,2 & 40,6 & 37,7 & 38 & 32,9 & 24,7 & 31,4 & 28,4 & 24 & 25,7 & 17,5 \\
\hline $\begin{array}{l}\text { Proportions de } \\
\text { députés écossais }\end{array}$ & 50,7 & 43,7 & 33,8 & 28,2 & 32,4 & 29,6 & 22,5 & 31 & 29,2 & 13,9 & 15,3 & 0 \\
\hline
\end{tabular}

\begin{tabular}{|l|l|l|l|l|l|}
\hline & 2001 & 2005 & 2010 & 2015 & 2017 \\
\hline PROPORTIONS DE SUFFRAgES & 15,6 & 15,8 & 16,7 & 14,5 & 28,6 \\
\hline PROPORTIONS DE DÉPUTÉS ÉCOSSAIS & 1,4 & 1,7 & 1,7 & 1,7 & 22 \\
\hline
\end{tabular}

Source : auteur

Tableau 2 : Résultats des Conservateurs écossais à divers scrutins

\begin{tabular}{|c|c|c|c|c|c|c|}
\hline & $\begin{array}{l}\text { Parlement } \\
\text { écossais } \\
2011\end{array}$ & $\begin{array}{l}\text { Collectivités } \\
\text { territoriales } \\
2012\end{array}$ & $\begin{array}{l}\text { Chambre } \\
\text { des } \\
\text { communes } \\
2015\end{array}$ & $\begin{array}{l}\text { Parlement } \\
\text { écossais } \\
2016\end{array}$ & $\begin{array}{l}\text { Collectivités } \\
\text { territoriales } \\
2017\end{array}$ & $\begin{array}{l}\text { Chambre } \\
\text { des } \\
\text { Communes } \\
2017\end{array}$ \\
\hline $\begin{array}{l}\text { Proportion } \\
\text { de } \\
\text { suffrages }\end{array}$ & 13,9 & 13,3 & 14,5 & 22 & 25,3 & 28,6 \\
\hline $\begin{array}{l}\text { Proportion } \\
\text { de sièges }\end{array}$ & 11,6 & 9,4 & 1,7 & 24 & 22,5 & 22 \\
\hline
\end{tabular}

Source : auteur 
Tableau 3 : Perception des leaders britanniques et écossais, mai 2017

\begin{tabular}{|l|l|l|l|l|l|}
\hline & Très bonne & Assez bonne & Assez mauvaise & Mauvaise & Indécis \\
\hline Ruth Davidson & 17 & 26 & 13 & 20 & 24 \\
\hline Theresa May & 9 & 26 & 22 & 30 & 13 \\
\hline Kezia Dugdale & 2 & 24 & 25 & 20 & 30 \\
\hline Jeremy Corbyn & 3 & 21 & 30 & 30 & 17 \\
\hline Nicola Sturgeon & 19 & 27 & 14 & 30 & 9 \\
\hline
\end{tabular}

Source : sondage YouGov, 15-18 mai 2017, www.yougov.co.uk, consulté le 13 octobre 2017. La question porte sur la personnalité en tant que leader d'un parti, ou chef de gouvernement pour May et Sturgeon

Tableau 4 : Répartition des résultats des candidats dans les circonscriptions (tranches de 10\%)

\begin{tabular}{|l|l|l|l|l|l|l|l|}
\hline & $<5 \%$ & $5-9,99 \%$ & $10-19,99 \%$ & $20-29,99 \%$ & $30-39,99 \%$ & $40-49,99 \%$ & $>50 \%$ \\
\hline Conservateurs 2015 & 1 & 23 & 22 & 9 & 4 & 0 & 0 \\
\hline Conservateurs 2017 & 0 & 1 & 18 & 20 & 16 & 3 & 1 \\
\hline Travaillistes 2017 & 0 & 3 & 13 & 12 & 27 & 3 & 1 \\
\hline $\begin{array}{l}\text { Libéraux-démocrates } \\
2017\end{array}$ & 46 & 4 & 3 & 1 & 3 & 2 & 0 \\
\hline SNP 2017 & 0 & 0 & 0 & 4 & 37 & 18 & 0 \\
\hline
\end{tabular}

Source : auteur

Tableau 5 : Dépenses des candidats Conservateurs (en proportion des plafonds autorisés) dans les circonscriptions ciblées en 2010 et en 2017

\begin{tabular}{|l|l|l|}
\hline & Élection 2010 & Élection 2017 \\
\hline Aberdeen South** $^{* *}$ & 98,1 & 92,83 \\
\hline Aberdeenshire West \& Kincardine** & 93,1 & 93,39 \\
\hline Angus** & 98,7 & 92,01 \\
\hline Argyll \& Bute & 95,5 & 94,24 \\
\hline Ayr, Carrick \& Cumnock** & 82,7 & 88,55 \\
\hline
\end{tabular}




\begin{tabular}{|l|l|l|}
\hline Ayrshire North \& Arran & 39,1 & 94,18 \\
\hline Banff \& Buchan** & 81,3 & 94,41 \\
\hline Berwickshire, Roxburgh \& Selkirk** & 98,9 & 64,51 \\
\hline Dumfries \& Galloway** & 99,2 & 92,39 \\
\hline Dumfriesshire, Clydesdale \& Tweeddale* & 98,2 & 90,67 \\
\hline East Lothian & 62 & 90,3 \\
\hline Edinburgh North \& Leith & 44,3 & 89,71 \\
\hline Edinburgh South West & 95,6 & 90,07 \\
\hline Gordon** & 41,6 & 94,29 \\
\hline Lanark \& Hamilton East & 21,2 & 91,28 \\
\hline Moray** & 86,3 & 94,23 \\
\hline Ochil \& South Perthshire** & 69 & 92,46 \\
\hline Perth \& Perthshire North** & 97,7 & 92,13 \\
\hline Renfrewshire East** & 97,5 & 93,42 \\
\hline Stirling** & 44,38 & 41,19 \\
\hline Écosse (moyenne) & & \\
\hline & & 93,22 \\
\hline
\end{tabular}

Source : calculs de l'auteur d'après Electoral Commission, 2017 UK Parliament Election. Candidates Spending, Londres, 2017.

Note : circonscriptions gagnées par les Conservateurs en $2005\left(^{*}\right)$ et en $2015(* *)$

Tableau 6 : Caractéristiques socio-démographiques des intentions de vote, mai 2017

\begin{tabular}{|l|l|l|l|l|l|l|}
\hline & $\begin{array}{l}18-24 \\
\text { ans }\end{array}$ & $\begin{array}{l}25-49 \\
\text { ans }\end{array}$ & $\begin{array}{l}50-64 \\
\text { ans }\end{array}$ & $\begin{array}{l}>65 \\
\text { ans }\end{array}$ & $\begin{array}{l}\text { Classe } \\
\text { moyenne }\end{array}$ & $\begin{array}{l}\text { Classe } \\
\text { ouvrière }\end{array}$ \\
\hline Conservateurs & 12 & 14 & 23 & 35 & 22 & 19 \\
\hline Travaillistes & 20 & 12 & 14 & 18 & 17 & 13 \\
\hline $\begin{array}{l}\text { Libéraux- } \\
\text { démocrates }\end{array}$ & 7 & 3 & 5 & 7 & 6 & 3 \\
\hline SNP & 38 & 35 & 30 & 24 & 31 & 32 \\
\hline
\end{tabular}

Source : sondage YouGov, 15-18 mai 2017, www.yougov.co.uk, consulté le 13 octobre 2017. 
Tableau 7 : Coefficients de corrélation du vote conservateur dans les circonscriptions : comparaisons entre 2015 et 2017

\begin{tabular}{|l|l|l|l|l|l|l|l|}
\hline & $\begin{array}{l}\text { Participation } \\
\text { même } \\
\text { élection }\end{array}$ & $\begin{array}{l}\text { Résultats } \\
\text { Conservateurs } \\
\text { précédion }\end{array}$ & $\begin{array}{l}\text { Dépenses } \\
\text { Conservateurs } \\
\text { même } \\
\text { élection }\end{array}$ & $\begin{array}{l}\text { Résultats } \\
\text { Travaillistes } \\
\text { même } \\
\text { élection }\end{array}$ & $\begin{array}{l}\text { Résultats } \\
\text { Libéraux- } \\
\text { démocrates } \\
\text { même } \\
\text { élection }\end{array}$ & $\begin{array}{l}\text { Résultats } \\
\text { SNP } \\
\text { même } \\
\text { élection }\end{array}$ & $\begin{array}{l}\text { Taux } \\
\text { chômage } \\
\text { premier } \\
\text { trimestre } \\
\text { année } \\
\text { élection }\end{array}$ \\
\hline $\begin{array}{l}\text { Conservateurs } \\
2015\end{array}$ & 0,34 & 0,92 & $\begin{array}{l}\text { Non } \\
\text { significatif* }\end{array}$ & $-0,42$ & $\begin{array}{l}\text { Non } \\
\text { significatif }\end{array}$ & $-0,47$ & $-0,48$ \\
\hline $\begin{array}{l}\text { Conservateurs } \\
2017\end{array}$ & 0,39 & 0,89 & 0,82 & $-0,62$ & $-0,2$ & $-0,28$ & $-0,38$ \\
\hline
\end{tabular}

Source : auteur

Note $\left(^{*}\right)$ : ce coefficient n'a pu être calculé en raison du caractère incomplet des données relatives aux dépenses publiées par la Commission électorale en 2015. En 2010, il s'élevait à 0,85 .

\section{BIBLIOGRAPHY}

Boffey, Daniel, « Losers no More. How the Tories Stopped Being Toxic in Scotland », The Observer, 8 mai 2016.

Convery, Alan, The Territorial Conservative Party. Devolution and Party Change in Scotland and Wales, Manchester, Manchester University Press, 2016.

Curtice, John, « Why No Tory Revival in Scotland? », in Torrance David (dir.), Whatever Happened to Tory Scotland ?, Édimbourg, Edinburgh University Press, 2012, p. 114-126.

Curtice, John, «The Three Characteristics of the Scottish Conservative Revival », http:// blog.whatscotlandthinks.org/2017/10/the-three-characteristics-of-the-scottish-conservativerevival/, consulté le 13 octobre 2017.

Davidson, Ruth, Conservative Party Conference Speech, http://blogs.spectator.co.uk/2017/10/ ruth-davidsons-conference-speech-full-text/, consulté le 13 octobre 2017.

Davidson, Ruth, « Reclaiming Liberal Democracy », The World in 2018, Londres, The Economist, décembre 2017, p. 58.

Deerin, Chris, « Ruth Davidson : "Brexit could Deliver a Hit we Can't Recover From" ", New Statesman, 6 septembre 2017.

Douglas-Hamilton, James, After You, Prime Minister, Londres, Stacey International, 2009. 
Edwards, Owen Dudley, « Election 2017 : the Promise of May », Scottish Affairs, vol. 26, n4, 2017, p. 478-494.

Electoral Commission, 2017 UK Parliament Election. Candidates Spending, Londres, 2017.

Gordon, Tom, « Ruth's Robotic Tendencies Face a Worrying Legal Challenge », The Herald, 24 juin 2017, p. 17.

Herald, « Davidson Breaks her Silence Over Two Conservative Trolls Readmitted into her Party », 25 août 2017, p. 1.

Jamieson, Bill (dir.), Scotland's Ten Tomorrows. The Devolution Crisis-and How to Fix It, Londres, Continuum, 2006.

Lang, Ian, Blue Remembered Years, Londres, Politico's, 2002.

Leask, David, « Only Two of Scotland's 13 Tory MPs went to Fee-paying Schools », The Herald, 17 juillet 2017, p. 6.

Macdonald, Catriona M.M. (dir.), Unionist Scotland 1800-1997, Édimbourg, John Donald, 1998.

Mitchell, James, Conservatives and the Union. A Study of Conservative Party Attitudes to Scotland, Édimbourg, Edinburgh University Press, 1990.

Monteith, Brian, Paying the Piper, Édimbourg, Birlinn, 2007.

Peterkin, Tom, «Ruth Davidson's Tories will Dare to Differ », Scotland on Sunday, 11 juin 2017.

Rifkind, Malcolm, Power and Pragmatism, Londres, Biteback, 2016.

Rifkind Commission, Scotland's Future, Édimbourg, 1998.

Strathclyde Commission, Made in Scotland, Édimbourg, 1998.

Scottish Conservatives, Commission on the Future Governance of Scotland, Édimbourg, mai 2014.

Scottish Conservatives, Building for Scotland. Strenghtening the Scottish Conservatives, Édimbourg, 2010.

Scottish Conservatives Commission, Scotland's Trading Future, Édimbourg, février 2017.

Scottish Conservative and Unionist Party, Forward, Together, Édimbourg, 2017.

Seawright, David, An Important Matter of Principle. The Decline of the Scottish Conservative and Unionist Party, Aldershot, Ashgate, 1999.

Settle, Michael, « Scottish Conservatives Set up New Think Tank to Boost Party Fortunes », The Herald, 4 octobre 2017, p. 8.

Settle, Michael, « Ruth Davidson's Intervention Forced May's Hand over Brexit Deal », The Herald, 6 décembre 2017, p. 1.

Smith, Alexander, Devolution and the Scottish Conservatives. Banal Activism, Electioneering and the Politics of Irrelevance, Manchester, Manchester University Press, 2011.

Strathclyde Commission, Made in Scotland, Édimbourg, 1998.

YouGov/The Times Survey Results, www.yougov.co.uk, consulté le 13 octobre 2017.

www.whatscotlandthinks.org consulté le 8 juin et le 22 décembre 2017. 


\section{NOTES}

1. David Seawright, An Important Matter of Principle, Aldershot, Ashgate, 1999, p. 200.

2. Ce terme, régulièrement employé auprès de journalistes (Daniel Boffey, « Losers no More. How the Tories Stopped Being Toxic in Scotland ", The Observer, 8 mai 2016), ne figure toutefois pas dans ses mémoires, où il est plutôt question de "faiblesses sérieuses ", Malcolm Rifkind, Power and Pragmatism, Londres, Biteback, 2016, p. 338.

3. Cet impôt local, par capitation, sans relation avec les capacités contributives, a été instauré par Thatcher en 1989, un an avant le reste du Royaume-Uni, à la demande des Conservateurs écossais, avant d'être aboli par Major en 1993.

4. C'était l'une des causes de leur déroute, James Mitchell, Conservatives and the Union, Édimbourg, Edinburgh University Press, 1990, p. 131. Cette position avait été imposée par Thatcher; son prédécesseur Edward Heath semblait plus ouvert. Dès 1999, les Conservateurs ont profité de l'élection à la représentation proportionnelle de 56 des 129 députés à Holyrood.

5. Strathclyde Commission, Made in Scotland, Édimbourg, 1998, p. 8-9. Lord Strathclyde avait été ministre au Scottish Office.

6. Scottish Conservatives, Building for Scotland, 2010, p. 16. Russell Sanderson, qui avait exercé diverses responsabilités au sein du parti écossais, était lui aussi entré à la Chambre des Lords pour devenir ministre au Scottish Office.

7. En 2000, aux débuts du Parlement écossais, les Conservateurs s'étaient opposés à la suppression de l'article 28 de la loi de 1986 interdisant aux enseignants des écoles publiques d'évoquer l'homosexualité comme relation familiale stable.

8. Cela contrastait avec la branche galloise, CONVERY Alan, The Territorial Conservative Party, Manchester, Manchester University Press, 2016, p. 115.

9. Scottish Conservatives, Commission on the Future Governance of Scotland, Édimbourg, 2014, p. 13.

10. Elle était populaire parmi les membres continuant à critiquer la dévolution, tel Monteith, député à Holyrood jusqu'en 2007. Brian Monteith, Paying the Piper, Édimbourg, Birlinn, 2007, p. 18. 11. SP, $O R, 26$ mai 2016, col. 14. Sur 31 députés conservateurs, deux (Jamie Green et Alison Harris) se sont abstenus, 7 autres (Maurice Corry, Gordon Lindhurst, Margaret Mitchell, Oliver Mundell, Gordon Simpson, Alexander Stewart et Ross Thompson) ont voté contre.

12. Sondages réalisés par Survation, Panelbase, BMG, YouGov et Ipsos-Mori, www.whatscotlandthinks.org, consulté le 8 juin 2017.

13. Tom Peterkin, «Ruth Davidson's Tories will Dare to Differ », Scotland on Sunday, 11 juin 2017.

14. Scottish Conservative and Unionist Party, Forward, Together, 2017, p. 6-7. Davidson a réussi le tour de force de fustiger la constance du SNP au regard de ses principes comme une inconstance, occultant ses propres accommodements avec son parti britannique. EDWARDS Owen Dudley, «Election 2017: The Promise of May », Scottish Affairs, vol. 26, n4, 2017, p. 493.

15. BMG Research, janvier et février 2017, www.whatscotlandthinks.org consulté le 8 juin 2017. 48-49\% des sondés refusaient ce second référendum avant le Brexit, $13 \%$ restant indécis.

16. Ils sont passés d'une volonté de garantir un accès maximal au marché unique, à une approche écossaise distincte, avant de se consacrer aux opportunités du Brexit pour l'ensemble du Royaume-Uni, SP, OR, 28 juin 2016 (Davidson, col. 16), 14 septembre 2016 (Jackson Carlaw, col. 29), 15 novembre 2016 (Fraser, col. 20), 17 janvier 2017 (Dean Lockhart, col. 22).

17. Scottish Conservatives Commission, Scotland's Trading Future, 2017, p. 21. Cette commission rassemblait des élus (parmi lesquels Tomkins) et des représentants des milieux d'affaires.

18. SP, OR, 25 avril 2017, col. 13.

19. SP, OR, 27 avril 2017, col. 54, Bill Bowman.

20. Scottish Conservative and Unionist Party, Forward, Together, 2017, p. 62-64. 
21. SP, OR, 25 avril 2017, col. 65, Fraser. Ils ont maintenu leur soutien jusqu'au vote final.

22. En 1992, ces sièges étaient Aberdeen South (est), Perth \& Kinross, Tayside North, Stirling, Édimbourg Pentlands et West, Eastwood, Ayr (centre), Dumfries, Galloway \& Upper Nithsdale (sud). Ce dernier avait été récupéré en 2001 et perdu en 2005. Avant 1987, les Conservateurs détenaient également, au nord-est, Angus, Moray, Banff \& Buchan, Kincardine \& Deesside et Gordon. Ils avaient aussi représenté Argyll \& Bute, Cunninghame North, North East Fife.

23. En 2000, seule la branche locale de South Ayrshire disposait encore d'une structure organisée. SMITH Alexander, Devolution and the Scottish Conservatives, Manchester, Manchester University Press, 2011, p. 55.

24. John Curtice, "The Three Characteristics of the Scottish Conservative Revival », http:// blog.whatscotlandthinks.org/2017/10/the-three-characteristics-of-the-scottish-conservativerevival/, consulté le 13 octobre 2017.

25. Auparavant, ils recueillaient les voix des opposants à la dévolution, CURTICE John, « Why No Tory Revival in Scotland», in David Torrance (dir.), Whatever Happened to Tory Scotland?, Édimbourg, Edinburgh University Press, 2012, p. 120.

26. David Leask, « Only Two of Scotland's 13 Tory MPs went to Fee-paying Schools », The Herald, 17 juillet 2017, p. 6. Au Parlement écossais, ils comptent encore un aristocrate, Alexander Burnett.

27. HC, Hansard, 2017, 25 juin (John Lamont), 26 juin (Andrew Bowie), 28 juin (Colin Clark, Ross Thomson), 29 juin (Luke Graham), 3 juillet (Bill Grant, Alistair Jack, Stephen Kerr, Paul Masterton), 17 juillet (Kirstene Hair), 12 septembre (Douglas Ross) et 16 novembre (David Duguid).

28. Clark, Jack, Kerr et Thomson.

29. HC, Hansard, 20 mars 2018., Duguid, Kerr, Duguid, Thomson, Lamont ont dénoncé la trahison de leurs administrés.

30. HC, Scottish Affairs Committee, 14 novembre 2017, Lamont, Duguid, Masterton, Thomson.

31. HC, Hansard, 17 octobre 2017, col. 276, Kerr.

32. HC, Hansard, 4 décembre 2017, col. 731, Masterton.

33. L'un d'eux, Ross, a préféré se rendre en Espagne pour arbitrer un match de football. HC, Hansard, 24 octobre 2017, col. 207.

34. HC, Hansard, 22 novembre 2017, col. 1052, Philip Hammond, Chancelier de l'Échiquier.

35. Chris Deerin, "Ruth Davidson: "Brexit could Deliver a Hit we Can't Recover From" ", New Statesman, 6 septembre 2017.

36. Michael Settle, «Ruth Davidson's Intervention Forced May's Hand over Brexit Deal », The Herald, 6 décembre 2017, p. 1.

37. SP, OR, 21 mars 2018, col. 157, Tomkins. Cet ex-membre de la commission Smith, qui siège à Holyrood depuis 2016, doutait de la constitutionnalité de la loi votée à l'initiative du gouvernement écossais.

38. SP, OR, 5 septembre 2017, col. 26.

39. SP, OR, 8 novembre 2017, col. 39, 19 décembre 2017, col. 27, Tomkins.

40. SP, OR, 21 février 2018, col. 64, Fraser. Celui-ci a été éclipsé par les Travaillistes dans sa tentative de dénonciation des hausses pour certains contribuables modestes et des coupes dans les budgets des collectivités territoriales. Une semaine plus tard, lors d'un débat informel sur la réforme de l'impôt local sur les personnes, peu progressif, les Conservateurs se sont prononcés contre sa suppression.

41. Le groupe est composé d'acteurs et d'observateurs de l'économie. SETTLE Michael, «Scottish Conservatives Set up New Think Tank to Boost Party Fortunes ", The Herald, 4 octobre 2017, p. 8.

42. Ruth Davidson, Conservative Party Conference Speech, http:// blogs.spectator.co.uk/2017/10/ruth-davidsons-conference-speech-full-text/ , consulté le 13 octobre 2017. 
43. Tom Gordon, «Ruth's Robotic Tendencies Face a Worrying Legal Challenge », The Herald, 24 juin 2017, p. 17. Parmi les raisons avancées figurent le manque d'investissement de Don au service du parti et des différends avec Davidson.

44. Herald, «Davidson Breaks her Silence Over Two Conservative Trolls Readmitted into her Party ", 25 août 2017, p. 1.

45. Soulignant que l'État et le marché ne sont pas inconciliables, elle prône un «capitalisme « compassionnel » dans une tribune («Reclaiming Liberal Democracy ») publiée par le numéro de prospective du magazine The Economist, The World in 2018.

46. Sondages YouGov et Survation, www.whatscotlandthinks.org, consulté le 22 décembre 2017. Selon les mêmes instituts, la tendance est comparable s'agissant des intentions de vote pour les élections au Parlement écossais.

\section{ABSTRACTS}

At the general election held on June 8 2017, the Scottish Conservatives who had only had one seat in the House of Commons since 2001 gained 13 MPs thanks to a twofold increase in their share of the vote. This recovery was put down to their leader Ruth Davidson. This article argues that although the latter's efforts should not be underestimated she has benefited from work undertaken under her predecessor and she has taken full advantage of the context, ie the resurgence of Scotland's constitutional issues with the prospect of Brexit. She now faces another challenge as her MPs will have to prove that they can defend Scottish interests.

Lors des élections à la Chambre des Communes du 8 juin 2017, les Conservateurs écossais, qui ne détenaient qu'un siège depuis 2001, en ont emporté 13 grâce à un doublement de leur part de voix. Cette victoire a été attribuée à leur leader, Ruth Davidson. Si celle-ci n'a pas ménagé ses efforts, elle a également bénéficié du travail entrepris par son prédécesseur, tout en tirant avantage du contexte, caractérisé par une résurgence des enjeux constitutionnels écossais dans la perspective du Brexit. Elle doit désormais relever un nouveau défi, afin de démontrer que ses députés sont capables de défendre les intérêts écossais.

\section{INDEX}

Mots-clés: Écosse, Conservateurs, Ruth Davidson, Chambre des Communes, indépendance écossaise, Brexit.

Keywords: Scotland, Conservatives, Ruth Davidson, House of Commons, independence, Brexit.

\section{AUTHOR}

\section{EDWIGE CAMP-PIETRAIN}

Laboratoire CALHISTE EA4343, Université de Valenciennes et du Hainaut-Cambrésis 